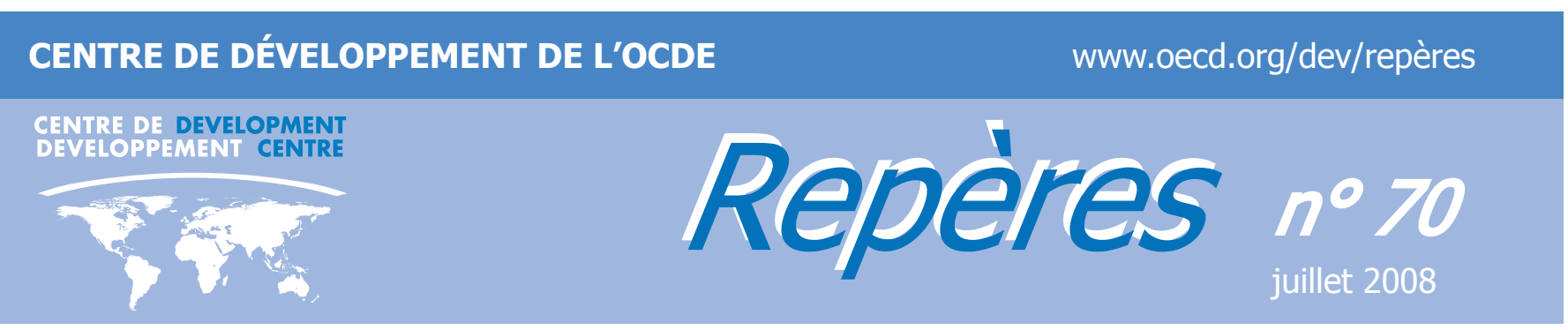

\title{
Les deux visages de l'emploi informel en Roumanie
}

par Denis Drechsler et Theodora Xenogiani, Centre de développement de I'OCDE

- L'emploi informel - extrêmement fréquent en Roumanie - freine son développement.

- Les politiques doivent viser deux groupes distincts : ceux qui quittent délibérément le système formel et ceux qui n'ont pas d'autre alternative.

- L'État doit faire évoluer l'attitude des populations à son égard et renforcer leur confiance dans les institutions.

En Roumanie, jusqu'à la moitié des emplưis écriappent à la législation du travail. Les travailleurs ne bénéficient que rarement d'une protection sociale ou d'avantages professionnels et les salaires payés au noir sont monnaie courante.

À ce niveau, le phénomène de l'informalité provoque de vraies difficultés. L'État perçoit moins de recettes fiscales et ne peut donc pas financer les infrastructures et les services publics. Quant aux travailleurs du secteur informel, qui ne bénéficient pas de filets de sécurité, ce sont les premières victimes des chocs économiques et de la pauvreté.

\section{Les causes de cette informalité}

On ne peut comprendre l'emploi informel en Roumanie sans mesurer sa diversité : le phénomène touche de nombreux secteurs - BTP, services et agriculture - et prend différentes formes - entreprises non immatriculées, travail soi-disant indépendant et sous-déclaration des revenus. Si certains choisissent délibérément de quitter le système formel, d'autres sont obligés de recourir au travail informel pour vivre.

\section{Figure 1. Les causes de l'emploi informel}

Poids des impôts

Sanctions inexistantse ou réduites

Programmes d'aide sociale

Conditions entourant les contrats

Procédures administratives

Piètre qualité des services publics

Coût des embauches / des licenciements

Niveau élévé du salaire minimum

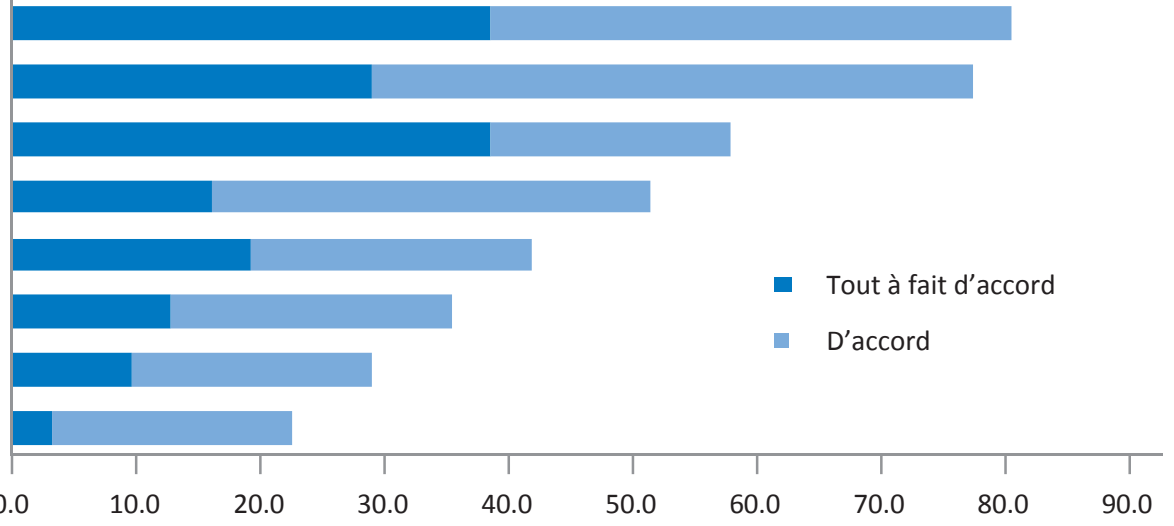




\section{À la recherche de politiques efficaces}

L'introduction d'un taux d'imposition unique en 2005 - inspirée par les résultats prometteurs obtenus en République tchèque - pourrait bien résorber l'emploi informel. Quant à la réduction des contributions sociales en 2003, elle semble avoir également eu des effets positifs. L'ensemble des initiatives visant à endiguer l'informalité sont, néanmoins, loin d'avoir été concluants.

D'autres mesures - comme l'augmentation du salaire minimum - ont à l'inverse apparemment réduit les offres formelles, notamment pour les groupes les plus défavorisés. Les récents programmes d'aide sociale, conçus spécifiquement pour les travailleurs informels, pourraient eux aussi avoir un effet contraire en confortant les incitations à rester dans le secteur parallèle. Ils pourraient même avoir conduit des travailleurs du secteur formel à en sortir - à l'instar de ce qui s'était passé au Mexique.

\section{Ouvrir les portes mais combler les brèches}

Les réformes, en Roumanie et ailleurs, doivent renforcer les avantages du secteur formel. Mais nombre d'entre elles restent inopérantes pour ceux qui n'ont pas d'autre choix que d'opter pour l'informalité. La solution passe par une approche différenciée entre ces travailleurs sans alternative et ceux qui quittent délibérément le secteur formel. La complexité de l'emploi informel doit impérativement être reconnue si l'on veut adopter une stratégie plus nuancée susceptible de répondre aux besoins spécifiques des travailleurs informels.
Des politiques volontaristes du marché du travail - comme la formation et les programmes de développement des compétences - contribueront à rouvrir les portes de la formalité. Les programmes d'aide sociale peuvent dépanner les populations n'ayant que peu d'options pour entrer sur le marché de l'emploi. Mais il faut aussi trouver le juste équilibre entre une sécurité sociale garantie et des incitations à rejoindre le marché formel du travail.

Quant à ceux qui ont choisi de quitter le secteur formel, il faut les inciter à y retourner. Les prestations devraient être liées aux niveaux de contributions sociales et les procédures administratives - comme l'immatriculation des entreprises et la déclaration des employés - devraient être simplifiées. La Roumanie doit également renforcer ses moyens d'application et de sanction.

\section{Un État crédible}

Aussi loin qu'aillent les politiques, elles ne remplaceront jamais la confiance. En Roumanie, l'informalité est avant tout l'expression d'une défiance à l'égard des institutions publiques, d'une perception négative du rôle de l'État et d'une mauvaise appréhension de l'intérêt de la sécurité sociale.

Pour obtenir une évolution durable, il va falloir modifier les attitudes et les croyances des gens. Des politiques plus novatrices - comme des campagnes d'information sur les avantages du travail formel et les dangers de l'informalité - pourront progressivement changer la donne. Le changement ne se fera pas en un jour, mais la Roumanie doit mettre les chances de son côté en adoptant dès à présent des politiques et des réglementations adaptées.

\section{Pour approfondir la question :}

Jante Parlevliet et Theodora Xenogiani, Report on Informal Employment in Romania, Working Paper No. 271, Centre de développement de l'OCDE, Paris.

Les lecteurs sont invités à citer ou reproduire les informations des Repères du Centre de développement de I'OCDE dans leurs propres publications. En échange, le Centre demande les remerciements de rigueur ainsi qu'un exemplaire de la publication. Le texte intégral des Repères et d'autres informations sur le Centre de développement et ses travaux sont disponibles sur : www.oecd.org/dev
Centre de développement de I'OCDE 2 , rue André-Pascal, 75775 Paris Cedex 16, France Tél : $33(0) 145.24 .82 .00$ Fax : $33(0) 144.30 .61 .49$ mél : cendev.contact@oecd.org 